\title{
Localized multiphoton photoactivation of paGFP in Drosophila wing imaginal discs
}

\author{
Periklis Pantazis* \\ Max Planck Institute of Molecular Cell Biology \\ and Genetics \\ Pfotenhauerstrasse 108 \\ 01307 Dresden, Germany
}

\author{
Marcos González-Gaitán \\ Max Planck Institute of Molecular Cell Biology \\ and Genetics \\ Pfotenhauerstrasse 108 \\ 01307 Dresden, Germany \\ and \\ Department of Biochemistry \\ Sciences II \\ 30 Quai Ernest-Ansermet, 1211 \\ Geneva 4, Switzerland
}

\begin{abstract}
In biological imaging of fluorescent molecules, multiphoton laser scanning microscopy (MPLSM) has become the favorite method of fluorescence microscopy in tissue explants and living animals. The great power of MPLSM with pulsed lasers in the infrared wavelength lies in its relatively deep optical penetration and reduced ability to cause potential nonspecific phototoxicity. These properties are of crucial importance for long time-lapse imaging. Since the excited area is intrinsically confined to the high-intensity focal volume of the illuminating beam, MPLSM can also be applied as a tool for selectively manipulating fluorophores in a known, three-dimensionally defined volume within the tissue. Here we introduce localized multiphoton photoactivation (MP-PA) as a technique suitable for analyzing the dynamics of photoactivated molecules with three-dimensional spatial resolution of a few micrometers. Short, intense laser light pulses uncage photoactivatable molecules via multiphoton excitation in a defined volume. MP-PA is demonstrated on photoactivatable paGFP in Drosophila wing imaginal discs. This technique is especially useful for extracting quantitative information about the properties of photoactivatable fusion proteins in different cellular locations in living tissue as well as to label single or small patches of cells in tissue to track their subsequent lineage. () 2007 Society of Photo-Optical Instrumentation Engineers. [DOI: 10.1117/1.2770478]
\end{abstract}

Keywords: green fluorescent protein; photoactivation; two-photon microscopy; Drosophila wing imaginal disc.

Paper 06280RR received Oct. 6, 2006; revised manuscript received Mar. 15, 2007; accepted for publication Mar. 16, 2007; published online Aug. 23, 2007.
The cloning of the green fluorescent protein (GFP) from the jellyfish Aequorea victoria ${ }^{1}$ and the subsequent successful engineering of a large variety of genetically encoded fluorescent tags (reviewed in Ref. 2) have revolutionized the way microscopy is used in biological research. Besides imaging the localization of fluorescent-tagged proteins or a fluorescent-marked cell population, it is possible to analyze their dynamics and to explore interactions between them in vivo (reviewed in Ref. 3). To determine the kinetic properties such as the diffusion of proteins of interest in vivo, their movements must be made visible. The most commonly used technique for this is fluorescence recovery after photobleaching (FRAP). ${ }^{4,5}$ With this method, a certain amount of tagged protein is rapidly bleached using a high-intensity laser pulse. The movement of the unbleached molecules from neighboring areas into the bleached area is then recorded by time-lapse microscopy.

However, with this method, fluorescence recovery involves the measurement of a small decrease in photobleached signal against a high background of unbleached fluorescence. This

\footnotetext{
*Present address: California Institute of Technology, Beckman Institute, Biological Imaging Center, MC 139-74,1200 E. California Blvd., Pasadena, CA 91125. Address all correspondence to Periklis Pantazis, Tel: 001-626-395-2863; Fax: 001-626-449-8599; E-mail: pantazis@caltech.edu; and Marcos GonzálezGaitán; Tel: 0041-22-379-6461; Fax: 0041-22-379-6470; E-mail: marcos.gonzalez@biochem.unige.ch
}

sensitivity disadvantage became obvious when the dynamics of the microtubules forming the mitotic spindle were examined using FRAP, which resulted in the failure to detect the slower directed motion of kinetochore microtubules possibly obscured by the much faster disassembly of a subset of microtubules. ${ }^{6,7}$ In addition, light of the same wavelength is used to photobleach the fluorophore and monitor at a reduced intensity the subsequent fluorescence recovery. Further photobleaching during monitoring is therefore inherent in this technique. A final drawback of FRAP is the requirement for high light intensity to photobleach GFP, increasing possible side effects of irradiation on biological systems like the emergence of reactive oxygen species.

An alternative to directly track cells in tissue or to follow the fate of individual molecules requires temporally and spatially selective activation. ${ }^{8}$ Thus, once activated, the mobility of the population of interest can be determined without background signal correction. Recently, a photoactivatable GFP (paGFP) has been described that can be used for such a labeling in live imaging. ${ }^{9}$ After intense illumination with 405-nm light, paGFP exhibits a 100-fold increase in 488-nm excited fluorescence. ${ }^{9}$ Using confocal laser scanning microscopy (LSM), paGFP has been demonstrated to be an ideal probe for monitoring temporal and spatial dynamics of chimerical pro-

1083-3668/2007/12(4)/044004/7/\$25.00 @ 2007 SPIE 

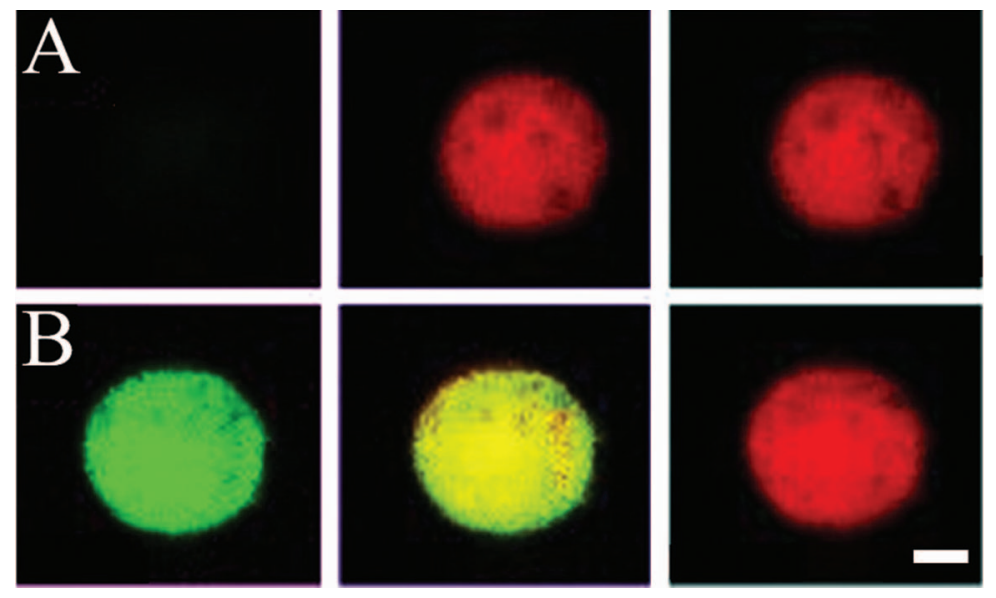

Fig. 1 Photoactivation of paGFP in Drosophila S2 cells. Photoactivation of paGFP was tested in vivo in Drosophila S2 cells co-transfected with cytosolic paGFP and DsRed. (a, b) Representative S2 cell co-transfected with cytosolic paGFP imaged under 488-nm excitation (left panels, green), DsRed imaged under 543-nm excitation (right panel, red) and overlays (center panel) prior (a) and after short illumination of the entire cell with 400-nm light (b). (a) Before photoactivation, very little fluorescence under 488-nm excitation is visible (left panel, green). (b) After photoactivation, the fluorescence signal of cytosolic paGFP under 488-nm excitation (left panel, green) increased in the entire cell. Fluorescence increases were determined by measuring the mean pixel values before and after photoactivation. Bar corresponds to $2 \mu \mathrm{m}$.

teins in vivo. ${ }^{10-12}$ For living samples, however, whose cells can be killed by the excitation of particularly ultraviolet and blue wavelength light, confocal microscopy may be a less preferred option for photoactivation. Furthermore, a spatially confined activation to allow the labeling of specific and sparse subpopulations of cells in the tissue to track their subsequent lineage or to monitor the dynamic of photoactivatable fusion proteins in different cellular locations (e.g., extracellular versus intracellular) is not possible.

Here, we introduce localized multiphoton photoactivation (MP-PA $)^{13}$ for uncaging photoactivated paGFP in defined cellular locations. Unlike confocal LSM, multiphoton excitation occurs only at the beam focus, resulting in spatially resolved photoactivation within the tissue that allows the tracking of movement of photouncaged proteins or cells. ${ }^{14}$ Because of the restricted excitation event, deleterious out-of-focus absorptions, photobleaching, and phototoxicity are reduced. The confinement of light-matter interaction due to multiphoton excitation makes MPLSM an ideal tool for selectively activating paGFP in a known, three-dimensionally defined volume within the tissue. Using short laser light pulses of 820-nm wavelength, we photoactivate cytosolic paGFP via twophoton excitation within single cells of Drosophila wing imaginal discs. Following subcellular photoactivation, this protocol offers the possibility to explore the dynamics of fusion proteins by tracking the photoactivated molecule that is the only visible GFP in the tissue. ${ }^{9}$

To analyze the characteristics of photoactivation-the rapid conversion of photoactivatable molecules to a green fluorescent state by intense illumination-we transfected cytosolic paGFP in Drosophila S2 cells. Briefly, Drosophila S2 cells ${ }^{15}$ were cultured in a standard Schneider medium. For the expression of cytosolic paGFP in S2 cells, they were transiently transfected with $3 \mu \mathrm{g}$ of DNA per well in 6 well plates using Cellfectin (Invitrogen) in serum-free medium. After 4 hours of incubation, cells were washed with PBS and wells were filled with $2 \mathrm{ml}$ of serum containing Schneider's medium. Cells were then kept in darkness for two days and were submitted to further analysis. To locate transfected cells, cotransfection with DsRed ${ }^{16}$ was performed. However, green fluorescence emitted by native paGFP when excited with low levels of $\sim 400$-nm was also sufficient to find positive cells (data not shown). The transfected cells were irradiated for several seconds with the light of a $100-\mathrm{W} \mathrm{Hg}^{2+}$ lamp using an excitation filter (D 395/40, AHF Analysentechnik AG) encompassing the major absorbance peak of native paGFP. ${ }^{9,17}$ Prior to photoactivation with $\sim 400-\mathrm{nm}$ light, paGFP displayed very little fluorescence at 488-nm excitation [Fig. 1(a)]. Upon photoactivation, fluorescence increased at least 50-fold at 488-nm excitation [Fig. 1(b)].

To test the feasibility of photoactivation in developing tissue, we activated cytosolic paGFP ubiquitously expressed under the control of the Actin promoter in Drosophila developing wing imaginal disc. In this case, photoactivation was performed in fixed tissue to allow precise spatial control of the activation event. Imaginal discs were dissected and mounted as previously described. ${ }^{18}$ The region of interest was irradiated using a $100-\mathrm{W} \mathrm{Hg}^{2+}$ lamp with a pinhole allowing photoactivation in a precise pattern (Fig. 2). In contrast to photoactivation of paGFP in Drosophila S2 cells where the cells were kept in darkness during the entire analysis, basal photoactivation prior to irradiation was visible throughout the tissue, probably caused by exposition to light during the wing imaginal dissection procedure. After illumination with $\sim 400$-nm light, the fluorescence increased up to at least 20fold when excited with 488-nm light. Once photoactivated, the absorbance and emission properties remained stable.

Directly tracking the lineage of distinct cell populations in tissue or monitoring the dynamics of molecules within single cells requires temporally and spatially selective activation of tagged proteins. To do this, we tested subcellular photoactivation with a confocal LSM using fixed Drosophila developing wing imaginal discs expressing paGFP. Optimal photoactivation was achieved with a pixel dwell time of less than $4 \mu \mathrm{s}$ and the maximum available laser power of $\sim 2 \mathrm{~mW}$ at the 


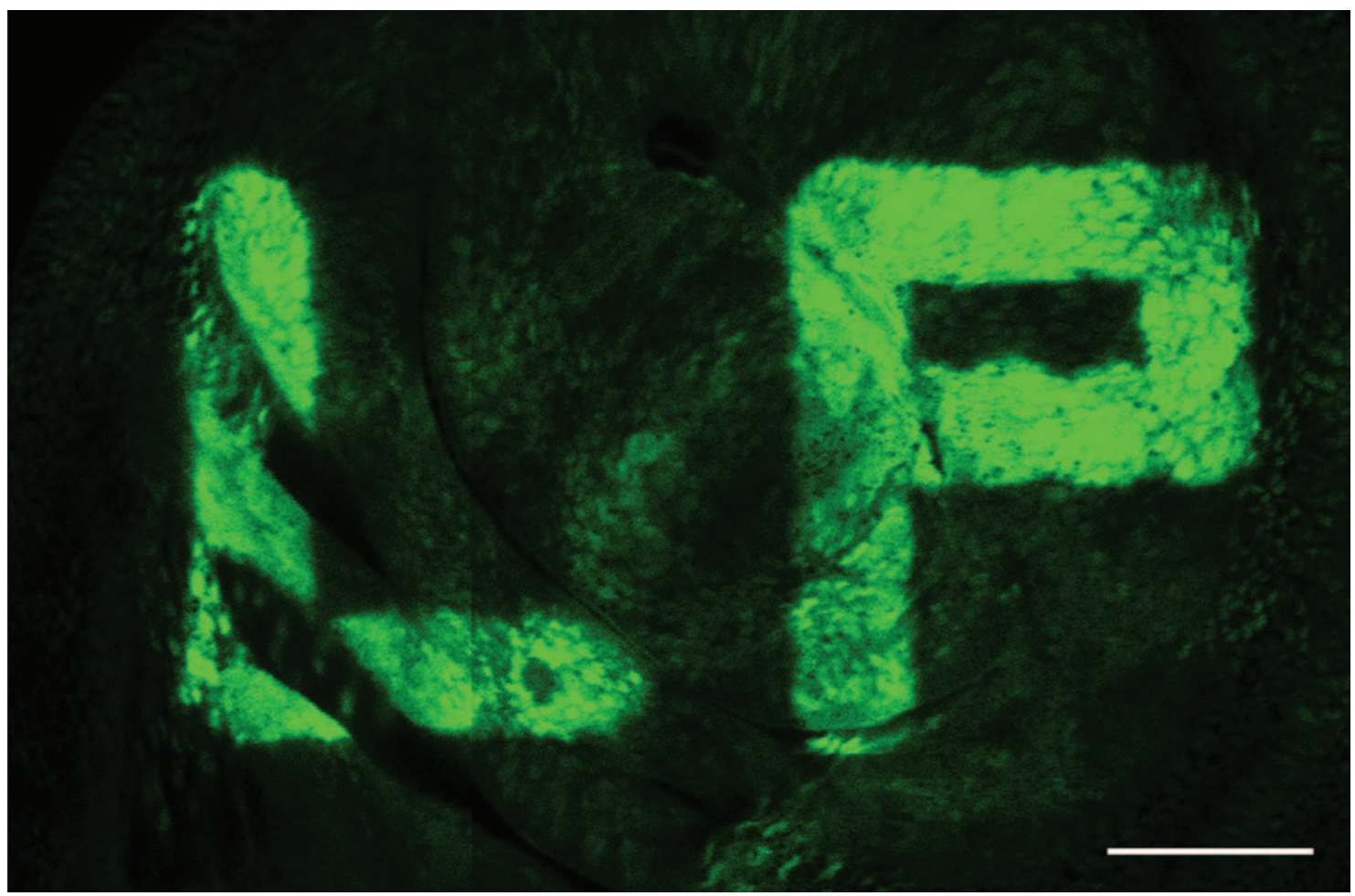

Fig. 2 Feasibility and precise spatial control of photoactivation of paGFP in a fixed Drosophila wing imaginal disc. Photoactivation of paGFP was performed in a fixed developing Drosophila wing expressing ubiquitously cytosolic paGFP under the control of the Actin promotor. Short illumination $(\sim 10 \mathrm{~s})$ with $\sim 400$-nm light photoactivated paGFP, increasing the fluorescence signal of cytosolic paGFP under 488-nm excitation in a precise pattern outlining the initials of the author's nickname "(Lakis)" and last name "(Pantazis)". Bar corresponds to $50 \mu \mathrm{m}$.

back aperture of a $40 \times 1.3$ numerical aperture (NA) PlanNeofluar objective (Zeiss). Longer exposure to 405-nm laser light as well as a change to a higher zoom factor decreased photoactivated paGFP fluorescence due to photobleaching. ${ }^{17}$ After illumination with high levels of 405-nm light, the fluorescence increased up to at least 20-fold for cytosolic paGFP when excited with 488-nm light (Fig. 3). The confocal LSM beam could activate cytosolic paGFP within a single cell (cell diameter of 3-4 $\mu \mathrm{m}$ ) [Fig. 3(a)]. However, this excitation event was not restricted to the focal plane. The $\mathrm{z}$-section through the Drosophila wing epithelium shows that the activation beam could not "select" an isolated slice within the tissue. An hourglass-shaped activation profile of cytosolic paGFP was visible that spanned over at least $2 \mu \mathrm{m}$ in the $x$-direction and over approximately $15 \mu \mathrm{m}$ in $z$-direction [Figs. 3(b) and 3(c)]. In summary, the confocal LSM cannot activate in defined cellular locations (apical versus basal or extracellular versus intracellular) that would allow tracking movement of photouncaged molecules.

In order to achieve a spatial isolated photoactivation event, subcellular photoactivation of paGFP in Drosophila tissue using two-photon LSM excitation was investigated. Due to the spatially restricted, nonlinear excitation probability, the activated volume was expected to have a finite thickness. ${ }^{13}$ Photoactivation of cytosolic paGFP expressed in Drosophila wing imaginal discs was performed on a Biorad Radiance 2100 MP two-photon setup attached to an Eclipse TE300 inverted microscope (Nikon) equipped with a $60 \times 1.2$ NA PlanApochromat objective (Nikon). Pulsed activation (pulse dura- tion $160 \mathrm{fs}$, repetition rate $80 \mathrm{MHz}$, average power after the objective $\leq 100 \mathrm{~mW}$ ) was provided by a 5 -W Verdi/Mira laser (Coherent). To avoid femtosecond pulse-induced tissue ablation, the activation conditions corresponded to peak intensities below or equivalent to $9 \times 10^{12} \mathrm{Wcm}^{-2}$ and to a pulse density (number of pulses received per surface unit in $\mu \mathrm{m}^{2}$ ) of approx. $10^{3} \mu \mathrm{m}^{-2}{ }^{19}$ Successful activation occurred at wavelengths between 750 and $830-\mathrm{nm}$ (data not shown), which is in good agreement with previous results. ${ }^{20}$ Under optimal peak intensities at 820-nm, cytosolic paGFP fluorescence also increased up to at least 20 -fold when excited with 488-nm (Fig. 4). Resembling confocal LSM, the two-photon LSM beam could activate cytosolic paGFP within a single cell [Fig. 4(a)]. In addition, this excitation event was limited to the focal plane. The cross-sectional view through the Drosophila wing epithelium shows that the activation beam could restrict the activation event to an isolated slice within the apical part of an epithelial cell in the tissue. Cytosolic paGFP was visible in a range of $1 \mu \mathrm{m}$ in the $x$-direction and spanned only over approximately $3 \mu \mathrm{m}$ in $z$-direction, which is well below the cell size of a single epithelial cell with a diameter of 3-4 $\mu \mathrm{m}$ and approximately $25 \mu \mathrm{m}$ in length [Figs. 4(b) and 4(c)]. In conclusion, the two-photon LSM can provide spatially resolved photoactivation events in defined cellular domains within a cell as well as excluding extracellular regions that would allow tracking movement of photouncaged molecules originated from defined intracellular regions. 

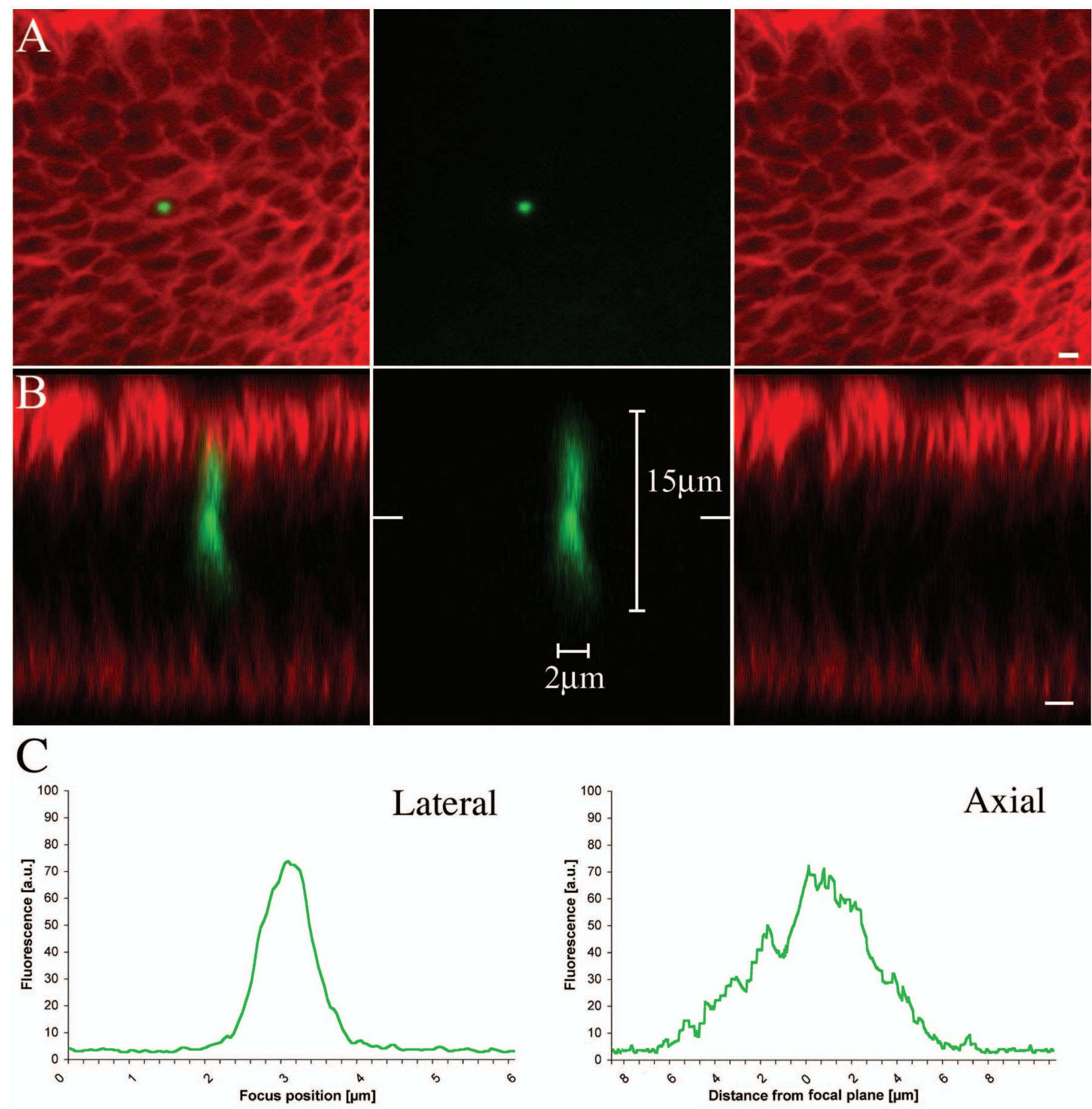

Fig. 3 Subcellular photoactivation of paGFP in a Drosophila wing imaginal disc with confocal LSM excitation. Subcellular photoactivation of paGFP with high levels of 405-nm light using confocal LSM excitation was tested in a fixed developing Drosophila wing expressing ubiquitously cytosolic paGFP under the Actin promotor. $(\mathrm{a}, \mathrm{b})$ Planar and axial view of the wing imaginal disc tissue double-stained showing activated paGFP fluorescence after photoactivation using confocal LSM excitation (center panels, green) and Fasciclin III immunostaining to label the cell profiles (right panels, red) and the overlays (left panels). (a) Photoactivated paGFP was imaged under 488-nm excitation and is visible within a single cell (center panel, green) in a Drosophila third instar wing disc tissue. (b) Axial view of the photoactivated region (center panel, green). Whereas a confocal LSM can activate cytosolic paGFP within a single cell, the cross-sectional view through the wing epithelium shows that the activation event is not restricted to the focal plane. (c) This result is illustrated by graphs of activated fluorescence versus lateral position (left graph) and axial position (right graph) at one level within the activated region. The lateral and axial resolution for confocal LSM activation are approx. $2 \mu \mathrm{m}$ and $15 \mu \mathrm{m}$, respectively. Bars correspond to $2 \mu \mathrm{m}$. 

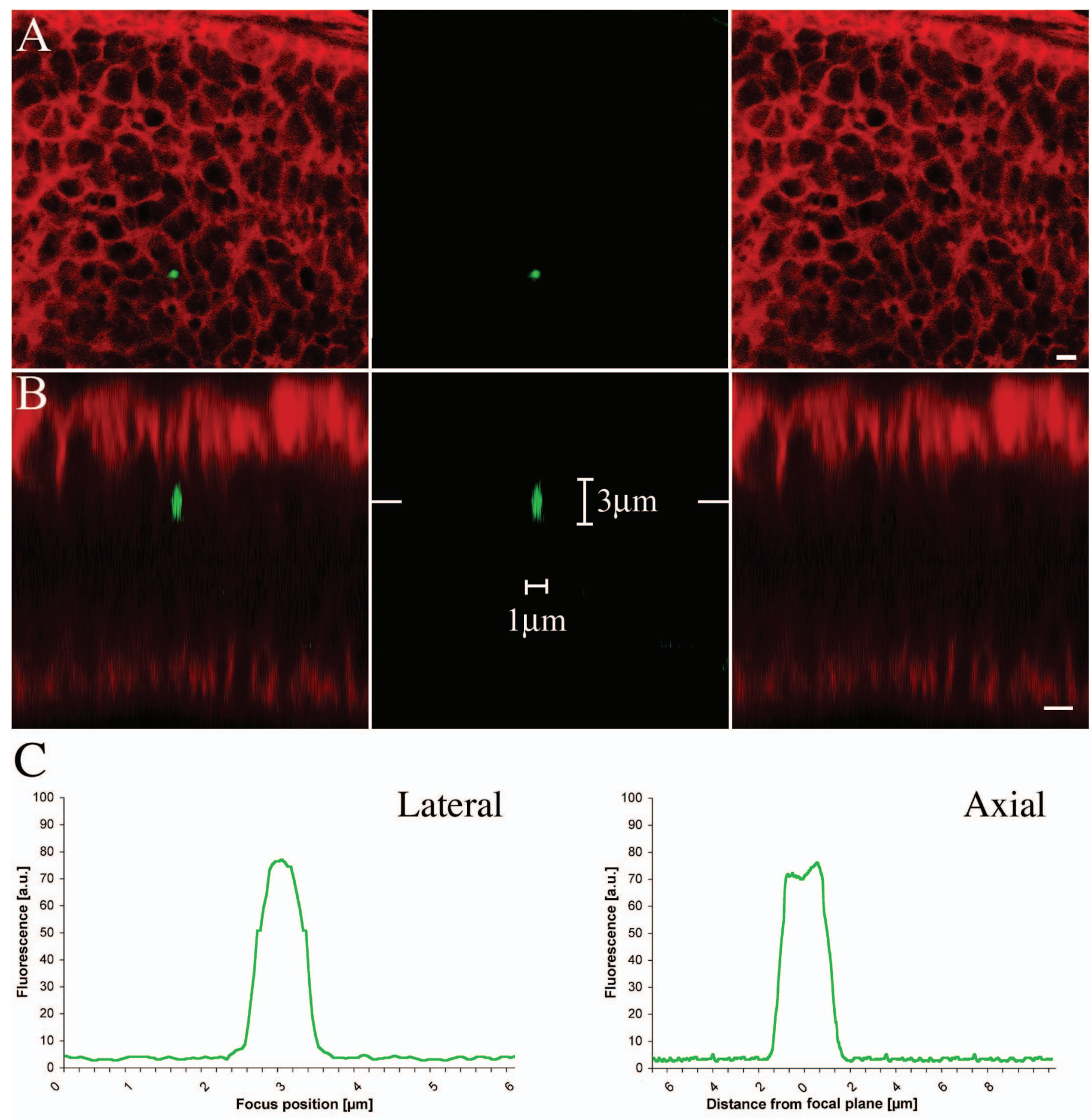

Fig. 4 Localized multiphoton photoactivation (MP-PA) of paGFP in a wing imaginal disc. Subcellular photoactivation of paGFP with 820-nm light using two-photon LSM excitation was tested in a fixed developing Drosophila wing expressing ubiquitously cytosolic paGFP under the Actin promotor. $(\mathrm{a}, \mathrm{b})$ Planar and axial view of the wing imaginal disc tissue double-stained showing activated paGFP fluorescence after photoactivation with a short pulse of 820-nm light using a two-photon LSM (center panels, green) and Fasciclin III immunostaining to label the cell profiles (right panels, red) and the overlays (left panels). (a) Photoactivated paGFP was imaged under 488-nm excitation and is visible within a single cell (center panel, green) in the Drosophila wing disc tissue. (b) Axial view of the photoactivated region (center panel, green). Note that a two-photon LSM can activate cytosolic paGFP within a single cell. In addition, the cross-sectional view through the wing epithelium shows that the activation event is approximately restricted to the focal plane. (c) This result is illustrated by graphs of activated fluorescence versus lateral position (left graph) and axial position (right graph) at one level within the activated region. The lateral and axial resolution for confocal LSM activation are approx. $1 \mu \mathrm{m}$ and $3 \mu \mathrm{m}$, respectively. Bars correspond to $2 \mu \mathrm{m}$. 
In this work we have demonstrated the use of MP-PA to selectively photoactivate paGFP in a three-dimensionally defined volume within an epithelial cell of the Drosophila wing imaginal disc. MP-PA differed significantly from confocal LSM photoactivation in the spatial extent of excitation. Unlike MP-PA, paGFP photoactivation using confocal LSM excitation occurred throughout the whole illumination cone that hit the sample. Importantly, since the activation rate of planes above and below the focal plane is equivalent to the activation rate in the focal plane, the total amount of photodamage to the tissue is significantly increased. In MP-PA, the excitation, and thus possible phototoxicity, was restricted to the focal volume of the objective. However, using peak intensities below $10^{13} \mathrm{Wcm}^{-2}$, we could not detect femtosecond pulse-induced tissue damage manifested usually in the onset of intense endogenous fluorescence at the scan point, and the possible occurrence of micro-explosions. ${ }^{19}$ Successful photoactivation ranged from 750 to $830-\mathrm{nm}$, which is in good agreement with previous results. ${ }^{20}$ Here, we have shown that paGFP can be photoactivated by diffraction-limited, two-photon pulsed laser excitation at $820-\mathrm{nm}$ that has been reported to be an optimal activation wavelength for cell viability in Drosophila tissue. ${ }^{12}$

The strength of MP-PA lies in the combination of high spatial resolution of the excitation event and the exploitation of photoactivation. Unlike FRAP, MP-PA generates a positive fluorescent signal against a negative background, which results in a more favorable signal-to-noise ratio. And in contrast to the observation of fluorescently tagged objects by constant imaging, the use of paGFP also allows the molecules of interest to be tracked without the need for continual visualization that greatly extends the spatio-temporal limits of biological dynamic studies and reduces the photobleaching and phototoxicity. This can be crucial when low levels of fluorescent labeling are required to prevent perturbation of the properties of the molecules of interest. With MP-PA, photoactivation of paGFP does not need intense ultraviolet illumination, requiring much less irradiation, which subsequently might reduce photodamage on the biological tissue. Moreover, using this protocol, precise fate-mapping experiments can be performed by labeling small patches of cells or even a single cell in different regions of a model organism, which, e.g., has proven difficult when analyzing the fate of heart precursor cells in zebrafish using single confocal LSM photoactivation of DMNB-caged fluorescein dextran. ${ }^{21}$

In addition, this protocol allows the direct extraction of kinetic properties of photoactivated proteins, since the precise origin of activated paGFP-tagged proteins is readily visible without the need for subtracting background fluorescence, as well as the discrimination of subcellular compartments by selective photoactivated labeling. Furthermore, the kinetics of optimal fluorescence intensity increase of paGFP is less than $1 \mathrm{~ms}$, allowing straightforward approaches to study rapid dynamics of protein behavior in cells or in tissue. Thus, once activated, the localization, the turnover, the direction as well as the mobility of the protein of interest can be determined in three dimensions. This is particularly useful when analyzing dynamic behaviors of subpopulations of, e.g., receptors, coreceptors, antagonists, and trafficking factors in living tissue. This way, endocytic, exocytic, recycling, and degradation rates can be measured both within a given cell (e.g., nucleus versus cytoplasm, growth cone versus axon, and apical versus basal endosomal populations) and between cells in living tissue (e.g., growth factors, cytokines, and extracellular matrix) and integrated into mathematical models to distinguish between different modes of movement or signaling. MP-PA will prove to be an excellent tool when addressing these questions.

\section{Acknowledgments}

We thank G. Patterson and J. Lippincott-Schwartz who kindly provided the paGFP-plasmid. We thank S.E. Fraser, A. Oates, V. Dudu, and N. Pantazis for critical reading and comments on the manuscript. We are indebted to K. Anderson and J. Peychl for assistance at the Biorad Radiance 2100 MP setup. We thank D. Backasch and A. Schwabedissen for technical assistance. This work was supported by the Max Planck Society, DFG, VW, and HFSP.

\section{References}

1. D. C. Prasher, V. K. Eckenrode, W. W. Ward, F. G. Prendergast, and M. J. Cormier, "Primary structure of the Aequorea victoria greenfluorescent protein," Gene 111, 229-233 (1992).

2. N. C. Shaner, P. A. Steinbach, and R. Y. Tsien, "A guide to choosing fluorescent proteins," Nat. Methods 2, 905-909 (2005).

3. J. Lippincott-Schwartz, E. Snapp, and A. Kenworthy, "Studying protein dynamics in living cells," Nat. Rev. Mol. Cell Biol. 2, 444-456 (2001).

4. D. Axelrod, D. E. Koppel, J. Schlessinger, E. Elson, and W. W. Webb, "Mobility measurement by analysis of fluorescence photobleaching recovery kinetics," Biophys. J. 16, 1055-1069 (1976).

5. D. E. Koppel, D. Axelrod, D. J. Schlessinger, E. L. Elson, and W. W. Webb, "Dynamics of fluorescence marker concentration as a probe of mobility," Biophys. J. 16, 1315-1329 (1976).

6. G. J. Gorbsky and G. G. Borisy, "Microtubules of the kinetochore fiber turn over in metaphase but not in anaphase," J. Cell Biol. 109 653-662 (1989).

7. E. D. Salmon, R. J. Leslie, W. M. Saxton, M. L. Karow, and J. R. McIntosh, "Spindle microtubule dynamics in sea urchin embryos: analysis using a fluorescein-labeled tubulin and measurements of fluorescence redistribution after laser photobleaching," J. Cell Biol. 99, 2165-2174 (1984).

8. J. C. Politz, "Use of caged fluorochromes to track macromolecular movement in living cells," Trends Cell Biol. 9, 284-287 (1999).

9. G. H. Patterson and J. Lippincott-Schwartz, "A photoactivatable GFP for selective photolabeling of proteins and cells," Science 297, 18731877 (2002)

10. Y. Shav-Tal, X. Darzacq, S. M. Shenoy, D. Fusco, S. M. Janicki, D. L. Spector, and R. H. Singer, "Dynamics of single mRNPs in nuclei of living cells," Science 304, 1797-1800 (2004).

11. L. Schermelleh, F. Spada, H. P. Easwaran, K. Zolghadr, J. B. Margot, M. C. Cardoso, and H. Leonhardt, "Trapped in action: Direct visualization of DNA methyltransferase activity in living cells," Nat. Methods 2, 751-756 (2005).

12. J. N. Post, K. A. Lidke, B. Rieger, and D. J. Arndt-Jovin, "One- and two-photon photoactivation of a paGFP-fusion protein in live Drosophila embryos," FEBS Lett. 579, 325-330 (2005).

13. W. Denk, J. H. Strickler, and W. W. Webb, "Two-photon laser scanning fluorescence microscopy," Science 248, 73-76 (1990).

14. M. Göppert-Mayer, "Über Elementarakte mit zwei Quantensprüngen," Ann. Phys. 9, 273-294 (1931).

15. I. Schneider, "Cell lines derived from late embryonic stages of Drosophila melanogaster," J. Embryol. Exp. Morphol. 27, 353-365 (1972).

16. M. V. Matz, A. F. Fradkov, Y. A. Labas, A. P. Savitsky, A. G. Zaraisky, M. L. Markelov, and S. A. Lukyanov, "Fluorescent proteins from nonbioluminescent Anthozoa species," Nat. Biotechnol. 17, 969-973 (1999)

17. G. H. Patterson and J. Lippincott-Schwartz, "Selective photolabeling of proteins using photoactivatable GFP," Methods 32, 445-450 (2004).

18. K. Kruse, P. Pantazis, T. Bollenbach, F. Julicher, and M. GonzalezGaitan, "Dpp gradient formation by dynamin-dependent endocytosis: 
Pantazis and González-Gaitán: Localized multiphoton photoactivation of paGFP...

Receptor trafficking and the diffusion model," Development $\mathbf{1 3 1}$ 4843-4856 (2004).

19. W. Supatto, D. Debarre, E. Farge, and E. Beaurepaire, "Femtosecond pulse-induced microprocessing of live Drosophila embryos," Medi. Laser Appl. 20, 207-216 (2005).
20. M. Schneider, S. Barozzi, I. Testa, M. Faretta, and A. Diaspro, "Twophoton activation and excitation properties of PA-GFP in the 720920-nm region," Biophys. J. 89, 1346-1352 (2005).

21. G. N. Serbedzija, J. N. Chen, and M. C. Fishman, "Regulation in the heart field of zebrafish," Development, 125, 1095-1101 (1998). 